\title{
Mean time to extinction of a metapopulation with an Allee effect
}

\author{
Chad E. Brassil \\ Department of Biology, University of Maryland, College Park, MD 20742, USA
}

\begin{abstract}
The incorporation of Allee effects into a simple metapopulation extinction model reveals a large non-linear reduction in mean time to extinction with small changes in an Allee limit. The extent of this reduction is dependent on the level of migration in the metapopulation. With small amounts of migration, small changes in the Allee limit result in large changes in the mean time to extinction. With higher levels of migration, the mean time to extinction is not as sensitive to changes in the Allee limit, becoming more similar to the single population case. The metapopulation modeled here is a set of nine patches, driven to extinction by environmental stochasticity. A generalized Allee effect is incorporated by a modification of the standard logistic model. The sensitivity of the mean time to extinction to small changes in the Allee limit is especially relevant to population viability analysis, which uses estimates of extinction times for management decisions. (C) 2001 Elsevier Science B.V. All rights reserved.
\end{abstract}

Keywords: Extinction; Migration; Metapopulation

\section{Introduction}

The term Allee effects has been used for many years to describe the phenomena of an increasing per capita growth rate with increasing density (Allee et al., 1949). This contrasts with normal density dependence in which the per capita growth rate decreases with increasing density. It is hypothesized that in most situations that exhibit an Allee effect, per capita growth rate increases initially at low densities and then decreases at higher densities due to normal density dependence. The literature describes a number of Allee effects (for review, see Dennis, 1989) which can be grouped into two general types. In cases of strong Allee effects, the per capita growth rate is actually negative at low densities, indicating more deaths than births in the population. A population exhibiting a strong Allee effect will have a lower density limit, below which the population deterministically goes toward extinction. A population exhibiting a weak Allee effect will have an increasing per capita growth rate at low densities, but will still be able to increase at low densities. In this study, only strong Allee effects will be considered.

Allee effect is a general term that can refer to a number of different specific phenomena. For example, an Allee effect can arise from the inability of mates to find each other at low densities, as has been demonstrated in Tribolium (Park, 1933). Allee effects can arise from the breakdown of a 
social structure at low densities. They can also arise from facilitative habitat modification of individuals within a population, for example, ascidians in which aggregations are better able to withstand water turbulence (Svane, 1984).

Metapopulations are systems of local populations connected by migration (Hanski and Gilpin, 1997). Classical metapopulation models described systems with extinction and recolonization of local patches (Levins, 1969). Recent work has begun to recognize the importance Allee effects might play in metapopulation dynamics. The incidence function model has been widely used to fit empirical presence/absence data into a metapopulation framework (Hanski and Thomas, 1994; Hanski, 1996). The assumption of weak Allee effects is incorporated into the derivation of the incidence function model, although the strength of the Allee effect cannot be manipulated by parameters in the equation (Hanski, 1994). This paper will examine changes in the scope of an Allee effect by changing the Allee limit.

A few studies have examined the impact of Allee effects on patch loss in metapopulations, finding an extinction threshold below which the metapopulation becomes extinct (Lande, 1987; Lamberson et al., 1992; Amarasekare, 1998; Groom, 1998). This paper will examine the impact of an Allee effect on the persistence time of a metapopulation with a fixed number of patches.

Environmental variation has been incorporated into metapopulation models, calculating the mean time to extinction (Mangel and Tier, 1993). Theoretical studies have attempted to find analytical solutions to mean extinction time of single populations for many years (Pielou, 1977; Nisbet and Gurney, 1982; Renshaw, 1991). Simulations can be used to find mean extinction times in more complex cases, using standard Monte Carlo techniques. Limited work has been done on mean extinction times of single populations experiencing an Allee effect (Stephan and Wissel, 1994).

\section{Model description}

Population dynamics in each patch were modeled using a modified version of the logistic equa- tion. An Allee limit $(l)$ is incorporated such that per capita population growth is negative below $l$. When $n>l$, the per capita growth rate is positive. As $n$ becomes large relative to $l$, the model approaches the standard logistic model (Wilson and Bossert, 1971).

$\frac{d n}{d t}=r n\left(1-\frac{n}{k}\right)\left(1-\frac{l}{n}\right)$

where $n$ is the population density, $t$ is time, $r$ is the growth rate and $k$ is the carrying capacity. For purposes of analyzing the effects of demographic stochasticity, the probability of a birth, $B(n)$, can be set to the positive terms of Eq. (1) and the probability of a death, $D(n)$, can be set to the negative terms of Eq. (1) (Nisbet and Gurney, 1982; Renshaw, 1991).

$$
\begin{aligned}
& B(n)=n r\left(1+\frac{l}{k}\right) \\
& D(n)=l r+\frac{n^{2} r}{k}
\end{aligned}
$$

In Eq. (2), the per capita death rate is density dependent and the per capita birth rate is density independent.

Environmental stochasticity was incorporated into Eq. (1) by adding a term $\varepsilon$ to the per capita growth rate. This term is a normally distributed random variable with zero mean (May, 1973; Hanski and Thomas, 1994).

$\frac{d n}{d t}=r n\left(1-\frac{n}{k}\right)\left(1-\frac{l}{n}\right)+n \varepsilon$

A nondimensional form of the equation was created for simplification of analysis by setting $N=$ $n / k, T=r t, L=l / k$ and $E=\varepsilon / r$ (Gurney and Nisbet, 1998; Murray, 1993).

$\frac{d N}{d T}=(1-N)(N-L)+N E$

A discrete analogue of Eq. (4) was utilized in the simulations, with a time step of 1 year.

$N_{t+1}=N_{t}+\left(1-N_{t}\right)\left(N_{t}-L\right)+N_{t} E$

if $L<N_{0}<1$, extinction of a patch can happen by the random occurrence of a large bad year, i.e. large negative $\varepsilon$, or by a consecutive series of 
small bad years, i.e. a series of small negative $\varepsilon$. Extinction can also occur with an overly large good year, i.e. a large positive $\varepsilon$, resulting in an overshoot of the carrying capacity, a depletion of resources and extinction the following year.

Migration $(M)$ was modeled as a stochastic process at each iteration. If a population exceeded the carrying capacity (i.e. $N>1$ ), then the following Eq. (6) was applied in each direction for possible immigrants.

$M_{t}=\mu N_{t}$

where $\mu$ is chosen from a truncated normally distributed random variable with zero mean and variance $\sigma^{2}$. If the randomly drawn value is negative, $\mu$ is set to zero. The metapopulation was constructed as a $3 \times 3$ grid, with migration between adjacent squares (Fig. 1). This grid was not adjusted, so any edge effects were fixed for all simulations. Eq. (6) was used for each connection, in each direction. Changes in the $\operatorname{var}(\mu)$, changes the variance in the amount of migrants that are exchanged between patches, but not the rate at which migrants are exchanged. Increases in $\operatorname{var}(\mu)$ also increases the maximum size of migrant propagules. Because the randomly chosen variable is truncated, increases in the variance simultaneously increase the mean number of migrants. Migration was manipulated by changing the variance, but because this also manipulated the mean number of migrants, I refer to changes in $\operatorname{var}(\mu)$ more vaguely as migration level. Migrant ex-

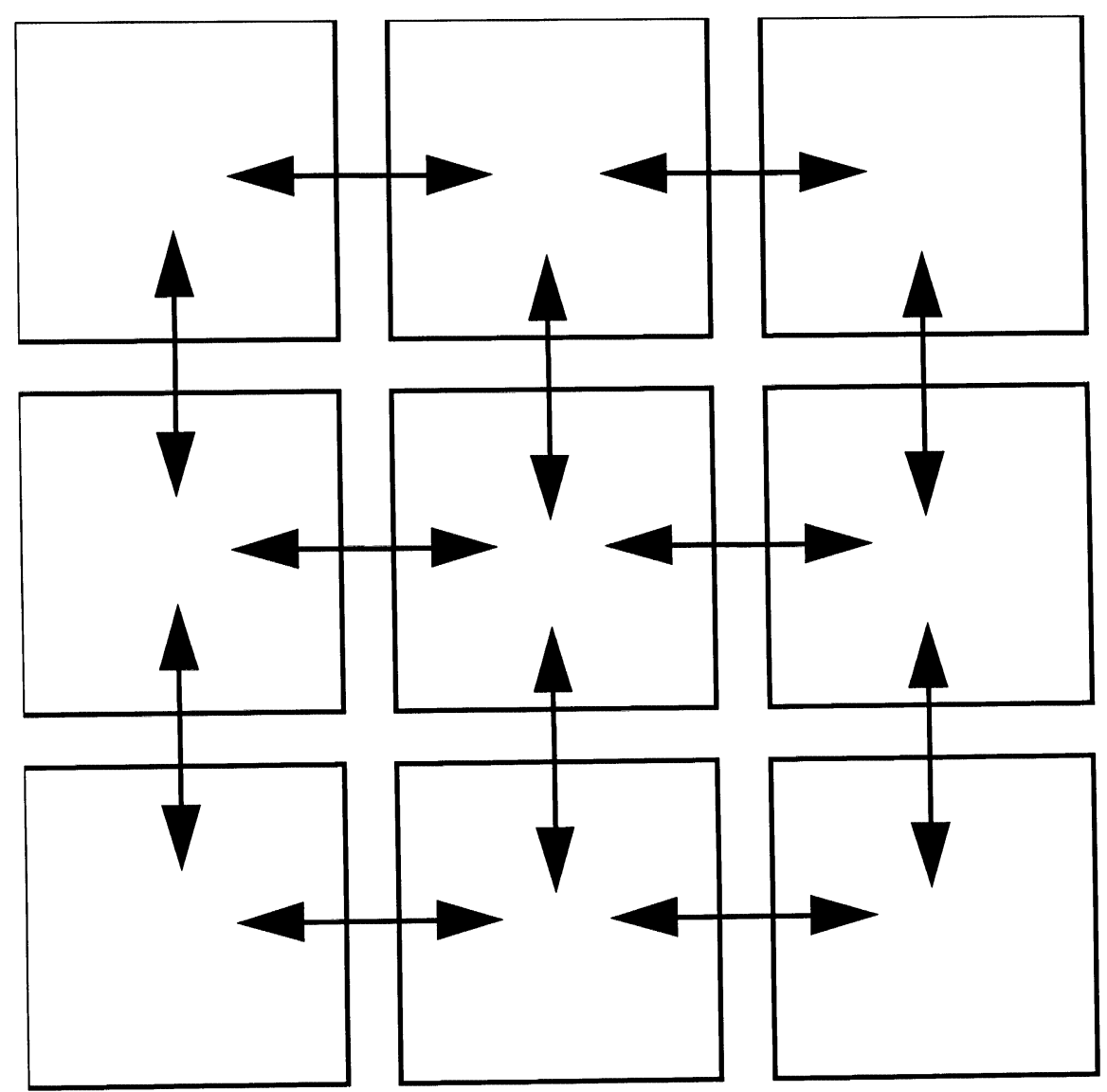

Fig. 1. Diagram of metapopulation structure used in this model. Patches of local population growth are shown by boxes. The arrows connecting patches represent possible migration connections. 


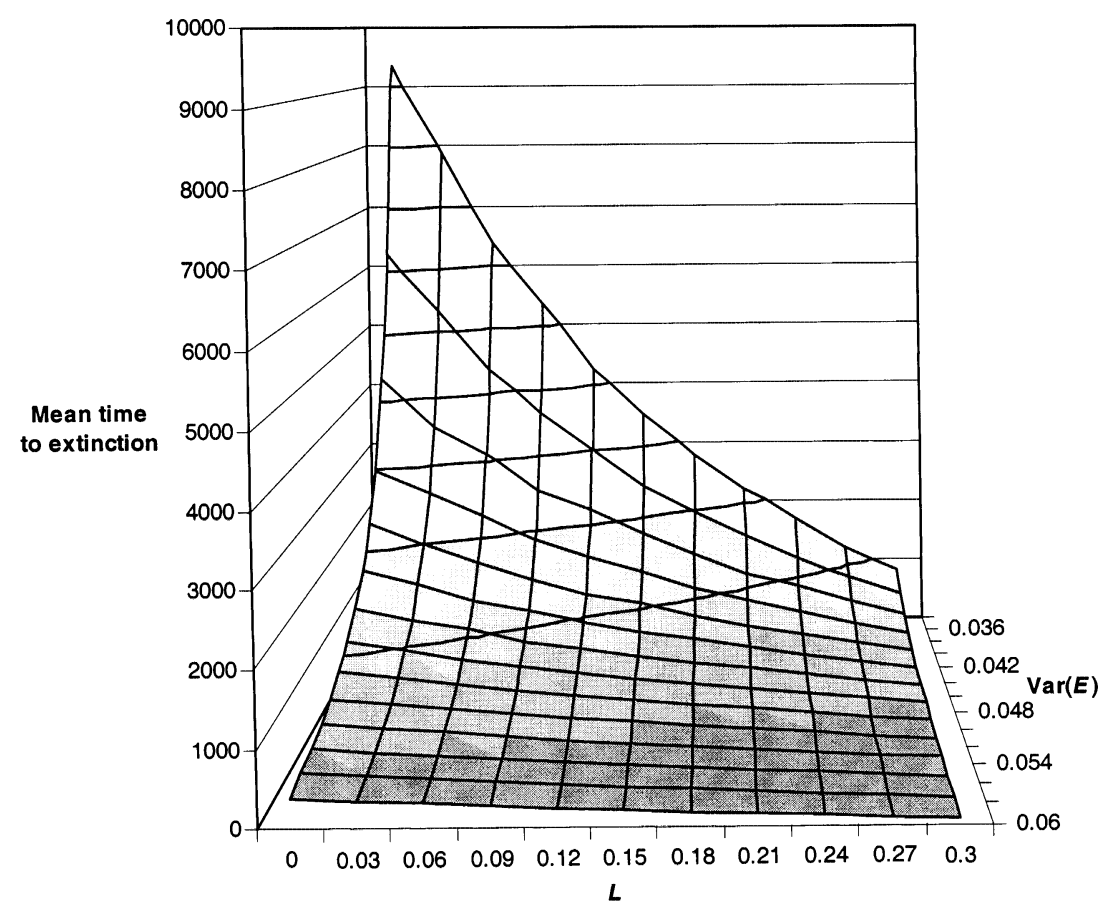

Fig. 2. Surface plot of mean time to extinction for a single population model. The scaled discrete model (Eq. (5)) was used in 10,000 simulations to calculate the geometric mean, plotted here versus environmental variance $(E)$ and $L$. $L$ is a scaled Allee limit which is directly proportional to the actual Allee limit $(l)$ and inversely proportional to changes in the carrying capacity $(k)$.

changes are calculated once during each iteration or once each year. In this model, there is no migration mortality.

The model was originally constructed in Stella Research 4.0. Simulations of single patch and metapopulation dynamics were conducted using $\mathrm{C}++$. The computer algorithms GASDEV and RAN1 were used to choose random numbers from a Gaussian distribution (Press et al., 1992). Unless noted, simulations consist of 10,000 replicate runs for each parameter set, with $N_{0}=0.5$ for each patch.

\section{Results}

The frequency distribution of extinction times was close to log-normal, so the geometric mean was used as a measure of central tendency.

The mean time to extinction of a single isolated patch decreases rapidly with increasing $\operatorname{var}(E)$, environmental variance or increasing $L$, the Allee limit scaled relative to the carrying capacity (Fig. 2 ). Increasing $L$ causes a greater reduction of the mean time to extinction in stable environments than in variable environments.

Changes in the Allee limit have similar effects when there is demographic stochasticity with no environmental stochasticity. Using the probabilities of birth and death in Eq. (2), the mean time to extinction was calculated using

$\sum_{i=1}^{\infty} \frac{B(1) B(2) \cdots B(i-1)}{D(1) D(2) \cdots D(i)}$

$+\sum_{m=1}^{n-1} \frac{D(1) D(2) \cdots D(i)}{B(1) B(2) \cdots B(i)} \sum_{i=m+1}^{\infty} \frac{B(1) B(2) \cdots B(i-1)}{D(1) D(2) \cdots D(i)}$

where $n$ is the initial population size (Nisbet and Gurney, 1982; Renshaw, 1991). A graph of the numerical solution for varying $l$ is given in Fig. 3 with $k=10, r=1.3$ and initial population of $n=$ 5. In this case, the mean time to extinction de- 
clines rapidly with increasing Allee limit, as it did under environmental stochasticity.

The mean time to extinction of a metapopulation with environmental stochasticity also decreases rapidly with increasing $\operatorname{var}(E)$ and $L$. As in the single patch model, increasing $L$ causes a greater reduction of the mean time to extinction in stable environments than in variable environments. However, because the metapopulation structure increases mean time to extinction compared to the single population, increased environmental variance was utilized in the simulations for manageable computation times. With increases in $L$, the mean time to extinction decreases more rapidly in the metapopulation than in the single patch model. Fig. 2 shows the response of mean time to extinction in a single patch over an increase in $L$ from 0 to 0.3 . Fig. 4 shows the response of a metapopulation to changes in $L$. Both figures demonstrate a similar shaped response, but the metapopulation shows this response over a much smaller $L$ interval, from 0 to 0.003 .

The metapopulation model has an additional migration parameter. With increases in migration, the mean time to extinction for the metapopulation increases. When $L>0$, a small amount of migration does not greatly increase the persistence. Migration must increase to the point of

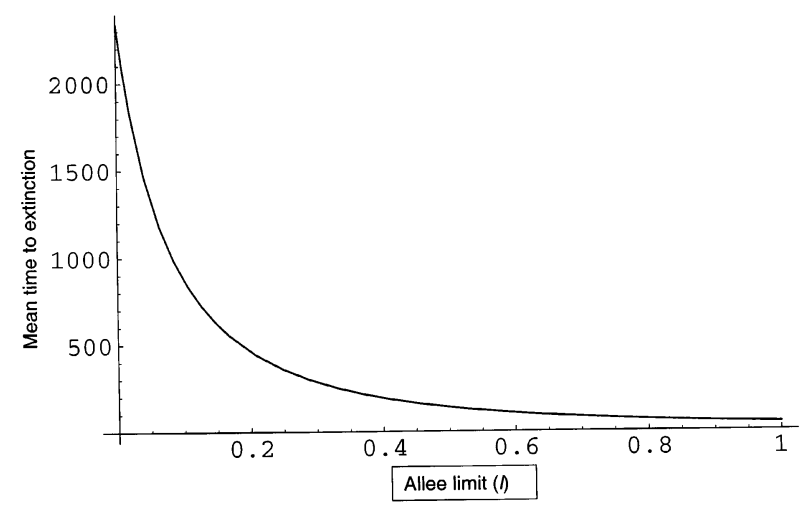

Fig. 3. The effect of Allee limit on mean time to extinction in a single population model with demographic stochasticity but no environmental stochasticity. The qualitative effect is similar to that under environmental stochasticity. Calculated using Eqs. (2) and (7), with $k=10, r=1.3$ and an initial population of $n=5$. overcoming $L$ before persistence time increases with increasing migration. At low levels of migration, not enough migrants arrive at a patch to overcome the Allee effect and the patch is not successfully recolonized. As migration is increased, this threshold is overcome at increasingly higher levels of $L$, until eventually all simulated levels of $L$ exhibit increasing extinction time with increasing migration. At high levels of migration, the relationship between mean time to extinction and $L$ approaches a $\log$-linear relationship. Fig. 5 illustrates the $\log$ (mean time to extinction) as a function of $L$ for various levels of migration variance.

In contrast to metapopulations with small amounts of migration, the relationship between mean time to extinction and $L$ for a set of disconnected patches $(\mu=0)$ is nearly linear. However, mean time to extinction is constrained to be greater than zero and the mean time to extinction at $L=0$ is much closer to zero in the disconnected patches. Therefore, it cannot be reduced as greatly by increasing $L$. The dashed line in Fig. 5 illustrates the relationship between mean time to extinction and $L$ for a set of disconnected patches with decreased environmental variance $(\operatorname{var}(E))$.

\section{Discussion and conclusions}

In a single population, increases in an Allee limit cause a non-linear decrease in mean time to extinction. The degree of this reduction is altered by a metapopulation structure. In a single population, to reduce the mean time to extinction by one-half, the scaled Allee limit needs to be increased from 0 to $0.12-0.18$. Biologically, this is a fairly significant Allee limit that would be readily detectable in a thorough field analysis of density dependent growth rates. The mean time to extinction of a metapopulation is much more sensitive to changes in the Allee limit. With a moderate migration level $(\operatorname{var}(\mu)=0.001)$, a small increase in the scaled Allee limit (from 0 to 0.003) is sufficient to halve the mean time to extinction. This relatively small Allee limit could easily be missed in a field analysis of density dependent growth rates with less migration, the metapopula- 


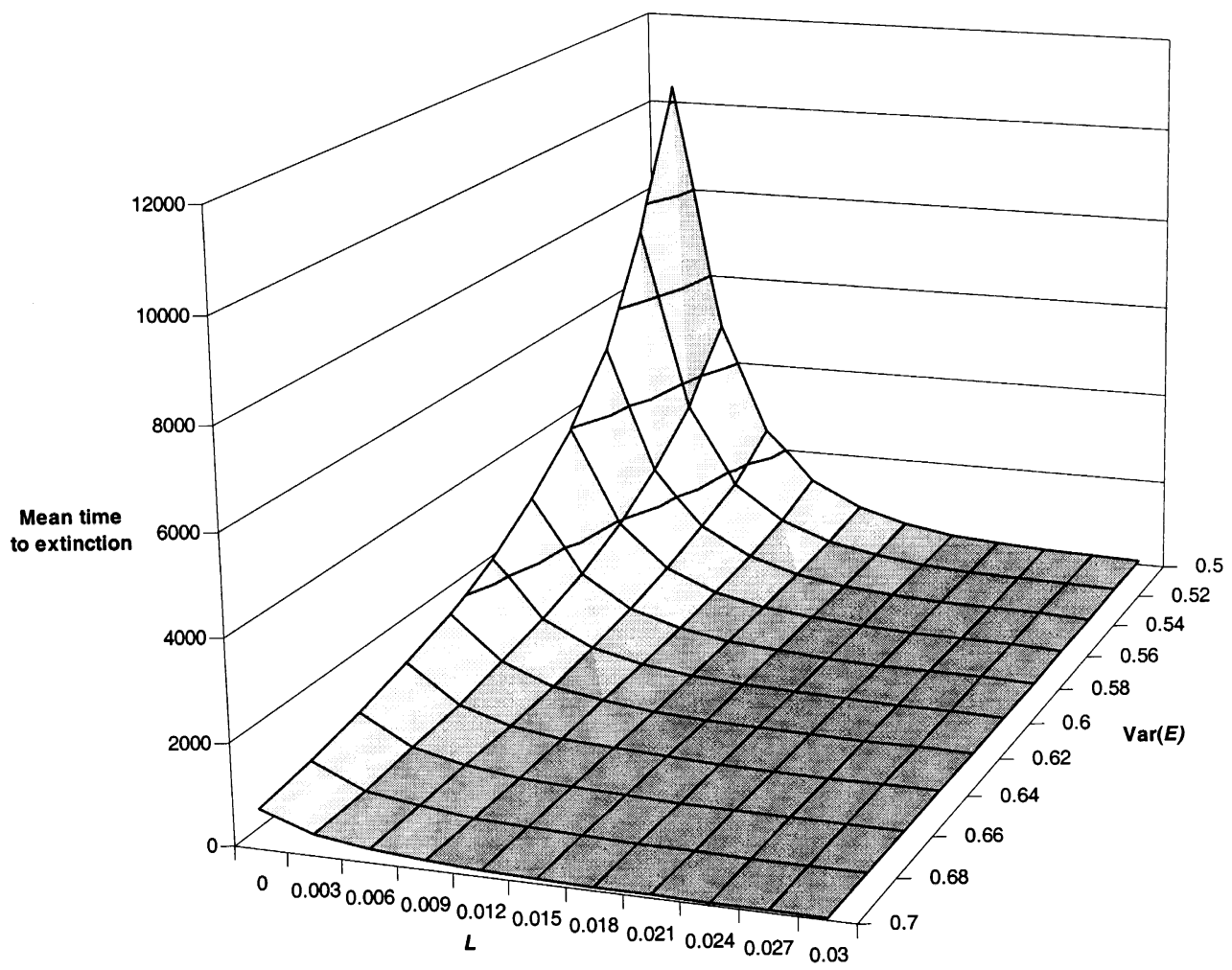

Fig. 4. Surface plot of mean time to extinction for a metapopulation model. Note that the scale of both environmental variance and $L$ are different in this figure compared to Fig. 3. The scaled discrete model (Eq. (5)) and the migration model (Eq. (6)) were used in 10,000 simulations to calculate the geometric mean, plotted here versus environmental variance $(E)$ and $L$. $L$ is a scaled Allee limit which is directly proportional to the actual Allee limit $(l)$ and inversely proportional to changes in the carrying capacity $(k)$. $\operatorname{Var}(\mu)=0.001$.

tion is even more sensitive to small changes in the Allee limit. Obversely, with high levels of migration and consequently high numbers of migrants re-colonizing patches, the results are more similar to the single species model.

Population viability analysis has been developed as a large body of literature which models extinction probabilities for management purposes (Groom and Pascual, 1998). Ignorance of an Allee effect could result in serious mis-estimation of population viability. This risk could be greatest in metapopulations with low environmental variation and low numbers of migration propagules.

Demographic stochasticity was not considered in combination with environmental stochasticity nor was it considered in the metapopulation model. It is generally believed that environmental stochasticity will dominate over demographic stochasticity in population dynamics (Nisbet and Gurney, 1982). However, with the small population sizes encountered in metapopulations, demographic stochasticity may be very important (Kendall, 1998), consequently modifying the predictions of this model.

The Allee limit was the only Allee effect parameter manipulated in this model. This is related to changes in the Allee effect scope, which I define as the range of population densities for which per capita growth rate increases with density, divided by the carrying capacity. The scope actually extends slightly higher than the Allee limit. Per capita growth rate positively increases at densities immediately above the Allee limit, until regular density dependence predominates 
and the model closely matches the standard logistic. Variation in the strength of the Allee effect was not considered. In all cases in this model, Allee effects were very strong, so that below the Allee limit, population growth was negative. The impact of weak Allee effects on mean time to extinction should be less pronounced than strong Allee effects. A number of basic Allee effect models have been proposed (Refs. in Gruntfest et al., 1997). However, none are set up to easily manipulate both strength and scope across all feasible ranges.

The general results of this model may not be applicable to systems in which migration operates differently than modeled here. In this model, the number of individuals which leave a patch is proportional to the number of individuals over carrying capacity which inhabit that patch. Migration decisions are unaffected by the state of neighboring patches. An animal that exhibits Allee effects may have evolved a mechanism to assess colonization success. For example, migration may only occur in groups large enough to ensure colonization success, or individuals may return to their original patch if they migrate to a sparsely populated patch. For example, models suggest that some Acorn Woodpeckers exist in a metapopulation structure (Stacey and Taper, 1992) and Acorn Woodpeckers, being social breeders, may even exhibit a form of an Allee effect. However, recent radio tracking of movement in Acorn Woodpeckers suggests that they visit many habitats throughout the season (Koenig et al., 1996), perhaps sampling habitat and local success. The model analyzed here was not designed to describe such adaptive movement. This model also does not examine the implications of patch heterogeneity. All patches are homogenous, with homogenous carrying capacity and homogeneous Allee limit.

Previously, single population models have shown that mean time to extinction increases more rapidly and in a more non-linear fashion with increases in the carrying capacity when environmental variation is small (Stephan and Wissel, 1994). When environmental variation is large, increasing the carrying capacity does little to increase mean time to extinction. This conclusion

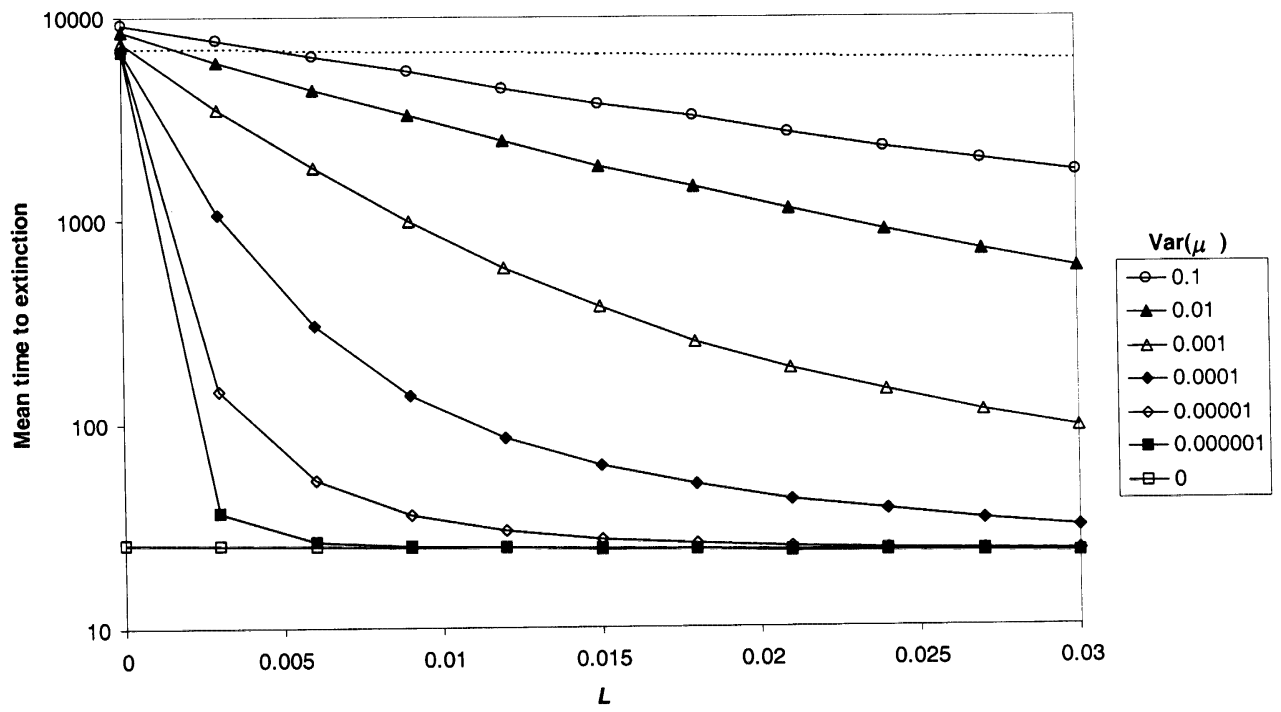

Fig. 5. Plot of mean time to extinction for a metapopulation model at different levels of migration. Note that the mean time to extinction is plotted on a logarithmic scale. The scaled discrete model (Eq. (5)) and the migration model (Eq. (6)) were used in 10,000 simulations to calculate the geometric mean, plotted here versus $L$. $L$ is a scaled Allee limit which is directly proportional to the actual Allee limit $(l)$ and inversely proportional to changes in the carrying capacity $(k)$. $\operatorname{Var}(E)=0.52$ for all solid lines. For comparison, the dashed line has $\operatorname{var}(\mu)=0$ and reduced environmental variance $(\operatorname{var}(E)=0.045)$. Both lines at var $(\mu)=0$ appear to have slope $=0$ in this graph, but actually have a slightly negative slope. 
can now be extended to include an Allee limit and migration level. In less variable environments, changes in the Allee limit have a greater, nonlinear effect. In a metapopulation, the effect becomes more non-linear. With reductions in the number of migrants, changes in the Allee limit or carrying capacity of the patches have an even greater, nonlinear effect.

\section{Acknowledgements}

I thank Peter Abrams for his thoughts and reviews, Robert Costanza and Alexey Voinov for their encouragement and three additional anonymous reviewers. This research was supported by the US Environmental Protection Agency's Science to Achieve Results Graduate Fellowship Program.

\section{References}

Allee, W.C., Emerson, A.E., Park, T., Schmidt, K.P., 1949. Principles of Animal Ecology. Saunders, Philadelphia.

Amarasekare, P., 1998. Allee effects in metapopulation dynamics. American Naturalist 152, 298-302.

Dennis, B., 1989. Allee effects: population growth, critical density and the chance of extinction. Natural Resource Modeling 3, 481-538.

Groom, M.J., 1998. Allee effects limit population viability of an annual plant. American Naturalist 151, 487-496.

Groom, M.J., Pascual, M.A., 1998. The analysis of population persistence: an outlook on the practice of viability analysis. In: Fiedler, P.L., Karieva, P.M. (Eds.), Conservation Biology, second ed. Chapman Hall, London, pp. 4-27.

Gruntfest, Y., Arditi, R., Dombrovsky, Y., 1997. A fragmented population in a varying environment. Journal of Theoretical Biology 185, 539-47

Gurney, W.S.C., Nisbet, R.M., 1998. Ecological Dynamics. Oxford University Press, New York.

Hanski, I., 1994. A practical model of metapopulation dynamics. Journal of Animal Ecology 63, 151-162.

Hanski, I., 1996. Metapopulation ecology. In: Rhodes, O.E., Chesser, R.K., Smith, M.H. (Eds.), Population Dynamics in Ecological Space and Time. University of Chicago Press, Chicago, pp. 13-43.
Hanski, I., Thomas, C.D., 1994. Metapopulation dynamics and conservation: a spatially explicit model applied to butterflies. Biological Conservation 68, 167-180.

Hanski, I., Gilpin, M.E., 1997. Metapopulation Biology: Ecology, Genetics and Evolution. Academic Press, San Diego, CA.

Kendall, B.E., 1998. Estimating the magnitude of environmental stochasticity in survivorship data. Ecological Applications 8, 184-193.

Koenig, W.D., Van Vuren, D., Hooge, P.N., 1996. Detectability, philopatry and the distribution of dispersal distances in vertebrates. TREE 11, 514-517.

Lamberson, R.H., McKelvey, R., Noon, B.N., Voss, C., 1992. A dynamic analysis of northern spotted owl viability in a fragmented forest landscape. Conservation Biology 6, 50512.

Lande, R., 1987. Extinction thresholds in demographic models of territorial populations. American Naturalist 130, 62435.

Levins, R., 1969. Some demographic and genetic consequences of environmental heterogeneity for biological control. Bulletin of the Entomological Society of America 15, 237-240.

Mangel, M., Tier, C., 1993. Dynamics of metapopulations with demographic stochasticity and environmental catastrophes. Theoretical Population Biology 44, 1-31.

May, R.M., 1973. Stability and Complexity in Model Ecosystems. Princeton University Press, Princeton, NJ.

Murray, J.D., 1993. Mathematical Biology. Springer, New York.

Nisbet, R.M., Gurney, W.S., 1982. Modelling Fluctuating Populations. Wiley, New York.

Park, T., 1933. Studies in population physiology. Journal of Experimental Zoology 65, 17-42.

Pielou, E.C., 1977. Mathematical Ecology. Wiley, New York.

Press, W.H., Teukolsky, S.A., Vetterling, W.T., Flannery, B.P., 1992. Numerical Recipes in C: The Art of Scientific Computing. Cambridge University Press, Cambridge.

Renshaw, E., 1991. Modelling Biological Populations in Space and Time. Cambridge University Press, Cambridge.

Stacey, P.B., Taper, M., 1992. Environmental variation and the persistence of small populations. Ecological Applications 2, 18-29.

Stephan, T., Wissel, C., 1994. Stochastic extinction models discrete in time. Ecological Modelling 75/76, 183-192.

Svane, I., 1984. Observations on the long-term population dynamics of the perennial Ascidian, Ascidia Mentula O.F. Muller, on the Swedish west coast. Biological Bulletin 167, 630-646.

Wilson, E.O., Bossert, W.H., 1971. A Primer of Population Biology. Sinauer Associates, Sunderland, MA. 